UK-04-08

MCTP-04-21

hep-th/0403275

\title{
Particle Production in Matrix Cosmology
}

\author{
Sumit R. Das ${ }^{a}$, Joshua L. Davis ${ }^{b}$, Finn Larsen ${ }^{b}$, and Partha Mukhopadhyay ${ }^{a}$ \\ ${ }^{a}$ Department of Physics and Astronomy \\ University of Kentucky, Lexington, KY-40506, U.S.A. \\ ${ }^{b}$ Michigan Center for Theoretical Physics \\ University of Michigan, Ann Arbor, MI-48109, U.S.A. \\ das@pa.uky.edu, joshuald@umich.edu, larsenf@umich.edu, partha@pa.uky.edu
}

\begin{abstract}
We consider cosmological particle production in $1+1$ dimensional string theory. The process is described most efficiently in terms of anomalies, but we also discuss the explicit mode expansions. In matrix cosmology the usual vacuum ambiguity of quantum fields in time-dependent backgrounds is resolved by the underlying matrix model. This leads to a finite energy density for the "in" state which cancels the effect of anomalous particle production.
\end{abstract}




\section{Introduction}

The spontaneous production of quantum particles in curved backgrounds is a profound phenomenon which has inspired progress in fundamental physics for several decades. In particular, cosmological particle production is, according to inflationary cosmology, the origin of all observable structure in the universe. It is clearly important to understand particle production better in a context where the quantum nature of gravity is taken fully into account. The purpose of this paper is to attempt this in the case of the matrix model for two dimensional string theory.

In the usual treatment of quantum fields in curved space-time cosmological particle production is the tangible consequence of an ambiguous vacuum: the vacuum state defined with respect to modes which are natural at early times typically contains particles when analyzed with respect to modes which are natural at late times (for a review see [1]). This is quite similar to Hawking radiation from black holes [2]. In string theory, holographic representations of gravity in terms of gauge theories have led to significant progress in our understanding of Hawking radiation $[3,4,5,6,7,8,9,10]$. In the gauge theory description, there is a preferred time and so a preferred vacuum; and Hawking radiation results arises through the normal quantum mechanical decay of a highly degenerate initial state $[4,6,11,12]$. It is natural to expect that string theory would provide a similar insight into the nature of cosmological particle production. This, however, has turned out to be rather difficult, particularly because of problems of formulating string theory in time-dependent backgrounds.

Recent progress in two dimensional non-critical string theory has improved the situation somewhat $[13,14]$. In this case there is a well understood holographic description - the matrix quantum mechanics of open strings - and also a closed string field theory, the two dimensional collective field theory of the eigenvalue density [15]. The holographic description has no space and has a unique time, while, in the closed string description, space arises from the space of eigenvalues $[15,16,17]$. Small fluctuations of the collective field then represent the perturbative states of the closed string theory (which in this case is a single massless scalar), while nontrivial time-dependent states, involving macroscopic numbers of decaying D0 branes, correspond to cosmological evolution. Such time dependent states can be studied unambiguously using the matrix model description.

The interpretation of time-dependent solutions in matrix theory as matrix cosmology was introduced recently by Karczmarek and Strominger [18] (the solutions themselves have been known for some time $[19,20,21])$. In the present paper we generalize their solutions by recognizing them as $W_{\infty}$ transforms of the ground state. This representation immediately allows the generation of several infinite families of cosmological solutions. Although our derivation is classical the solutions clearly exist at the quantum level.

The fluctuations around the cosmological backgrounds are efficiently described by the col- 
lective field theory of the matrix eigenvalues. The fluctuating field in fact reduces to a massless scalar field in two dimensions, a popular toy model for studying vacuum ambiguities. The novelty in the present context is that the vacuum state of the scalar field is inherited from the ultraviolet completion of the theory, i.e. the matrix model. This means the short-distance divergences of the energy-momentum tensor are cancelled, rather than subtracted as in usual quantum field theory. It also means a specific, finite, energy is associated with the static vacuum, namely the energy which, in the matrix model, is the standard one loop energy of the ground state. In matrix cosmology this ground state energy will contribute a novel timedependent term in the EM-tensor which happens to precisely cancel the usual contribution from particle production. The VEV of the EM-tensor is thus identical in the initial and final states of the cosmology, a highly unusual situation.

It is often confusing what the correct observables are in time-dependent string theory. This concern seems particularly acute in matrix cosmology where the universe tends to have dramatic initial and/or final conditions, such as the complete disappearance of space-time at early or late times. In the context of quantum field theory the problem with such non-adiabatic evolution is that the particle concept is not useful. In matrix cosmology there is a preferred vacuum, i.e. a notion of no particle state which is universal, and applicable even as space-time is disappearing. This enable us to discuss cosmological particle in a setting that would be difficult to analyze using ordinary quantum field theory.

This paper is organized as follows. In section 2 we introduce the cosmological solutions to the matrix-model. In section 3 we first discuss the causal structure and the observables of matrix cosmology. We then construct the explicit modes of the fluctuating quantum fields and deduce the corresponding Bogulubov coefficients for particle production. Finally, in section 4, we compute the energy-momentum tensor of the model, using anomalies.

Note added: as this paper was being prepared we received [22] which has some overlap with the present work, particularly section 3. However, the main points of the works are different.

\section{Matrix Cosmology}

In this section we introduce the matrix cosmologies of Karczmarek and Strominger [18], along with our generalizations. We discuss in turn the matrix model description, the collective field theory, and the fluctuations in collective field theory.

\subsection{The Fermion Phase Space Picture}

The holographic description of two dimensional closed string theory is in terms of singlet states of the quantum mechanics of a single $N \times N$ hermitian matrix $M$ with some invariant potential 
$\operatorname{Tr} V(M)$ which has a quadratic maximum. We will choose the value of $V(M)$ at this maximum to be zero. The dynamics can be entirely recast in terms of the eigenvalues $x_{i}(t)$ of the matrix $M$ and interpreted as $N$ fermions with positions $x_{i}(t)$ in an external potential $V(x)$. The double scaling limit consists of tuning the coupling constants involved in the potential and taking $N \rightarrow \infty$ so that the Fermi energy $-\mu_{F} \rightarrow 0$ while the rescaled Fermi energy $\mu=\beta N \mu_{F}$ is held fixed. In this limit the coordinates and the momenta of the fermions may be rescaled such that the single particle Hamiltonian becomes the inverted harmonic oscillator

$$
h=-\frac{1}{2} \frac{d^{2}}{d x^{2}}-\frac{1}{2} x^{2}
$$

The Fermi energy of this rescaled problem is $-\mu$. Interpreting the double scaling limit as a continuum limit of string world-sheets one can identify the corresponding string coupling as $g_{s}=\frac{1}{\mu}$.

In the classical limit we can discuss the dynamics in terms of fermion trajectories in phase space $(x, p)$. In the ground state, the Fermi surface is

$$
\frac{1}{2} p^{2}-\frac{1}{2} x^{2}=-\mu
$$

which implies that all states with $p^{2}<x^{2}-2 \mu$ are filled. In this paper we will concentrate on the region $x<0$. As is known now, the excitations of the Fermi sea in the $x>0$ region simply represents a second massless scalar present in the 0B theory [23].

The action corresponding to (1) has an infinite symmetry algebra, $W_{\infty}[24,25,26]$, whose generators are given by

$$
w_{r s}=\frac{1}{2} e^{(r-s) t}(x-p)^{r}(x+p)^{s} .
$$

The Hamiltonian is one of these charges, $h=-w_{11}$. At the classical level the charges $w_{r s}$ satisfy the Poisson bracket algebra

$$
\left\{w_{r s}, w_{r^{\prime} s^{\prime}}\right\}_{P B}=\left(r s^{\prime}-s r^{\prime}\right) w_{r+r^{\prime}-1, s+s^{\prime}-1}
$$

Our interest in $W_{\infty}$ as that it generates nontrivial solutions: starting with any classical solution, a new classical solution can be found by transforming with an $W_{\infty}$ element. In particular a static Fermi sea like (2) can be transformed into a time dependent Fermi sea by using charges with $r \neq s$. We will concentrate on solutions generated in this way by charges $w_{r 0}$ and $w_{0 s}$. For these the finite transformations of $x$ and $p$ become

$$
\begin{array}{lll}
w_{0 s}: & x^{\prime}=x+\lambda s e^{-s t}(x+p)^{s-1}, & p^{\prime}=p-\lambda s e^{-s t}(x+p)^{s-1} . \\
w_{r 0}: & x^{\prime}=x+\lambda r e^{r t}(x-p)^{r-1}, & p^{\prime}=p+\lambda r e^{r t}(x-p)^{r-1}
\end{array}
$$


where $\lambda$ is the parameter of transformation. Thus, starting from the static Fermi surface (2), we can obtain an infinite set of exact solutions characterized by Fermi surfaces

$$
\begin{aligned}
& w_{0 s}: \frac{1}{2}\left(x^{2}-p^{2}\right)+\lambda s e^{-s t}(x+p)^{s}=\mu . \\
& w_{r 0}: \frac{1}{2}\left(x^{2}-p^{2}\right)+\lambda r e^{+r t}(x-p)^{r}=\mu .
\end{aligned}
$$

Of particular interest are the solutions obtained by the actions of $w_{01}$ and $w_{10}$. In these cases the $W_{\infty}$ transformations (5-6) reduce to conventional coordinate transformations which are simply time dependent shifts of the coordinate. It is then clear that we can create a twoparameter family of solutions by combining the transformations $w_{01}$ and $w_{10}$ with arbitrary parameters

$$
\begin{aligned}
& x^{\prime}=x+\lambda_{-} e^{-t}+\lambda_{+} e^{t} \\
& p^{\prime}=p-\lambda_{-} e^{-t}+\lambda_{+} e^{t}
\end{aligned}
$$

The resulting solutions

$$
\frac{1}{2}\left(x^{2}-p^{2}\right)+\lambda_{-} e^{-t}(x+p)+\lambda_{+} e^{t}(x-p)=\mu,
$$

are in fact the solutions discussed by Karczmarek and Strominger [18] (up to a redefinition of the string coupling constant).

The more general cosmological solutions given by (8) also correspond to smooth Fermi surfaces. A nice way to map them is to introduce the coordinates $x_{ \pm}=x \pm p$ in phase space. Then the solutions generated by $w_{0 s}$ take the form

$$
x_{-} x_{+}+2 \lambda s e^{-s t} x_{+}^{s}=2 \mu .
$$

We will consider the case of $\lambda>0$. After the further rescalings $y_{-}=x_{-} / 2 \mu \alpha$ and $y_{+}=\alpha x_{+}$ with the time-dependent factor

$$
\alpha=\left[\frac{\lambda s}{\mu}\right]^{1 / s} e^{-t}
$$

the Fermi surface becomes

$$
y_{-}=\frac{1}{y_{+}}\left(1-y_{+}^{s}\right) .
$$

Interestingly this surface is symmetric around $y=0$ in phase space only for even $s$, since only then $y_{-}\left(-y_{+}\right)=-y_{-}\left(y_{+}\right)$. Concentrating as usual on the region in which $y_{+}<0$ we find, for odd $s$ a smooth Fermi surface interpolating between $y_{-} \sim 1 / y_{+}$for small $y_{+}$and $y_{-} \sim y_{+}^{s-1}$ for large $y_{+}$. Importantly, since the quantity $\frac{d y_{-}}{d y_{+}}$vanishes only once in this domain, there are no "folds" in the Fermi surface. This means it can be characterized by its intersection with a $x_{+}=$constant line, i.e. by the function $x_{-}=x_{-}\left(x_{+}\right)$. For even $s$, there is no zero of $\frac{d y_{-}}{d y_{+}}$and 
the Fermi surfaces actually cross over to positive values of $y_{-}$. In this description the Euler equations for the Fermi surface are simply

$$
\partial_{t} x_{ \pm}= \pm x_{ \pm} \pm x_{\mp} \frac{\partial x_{ \pm}}{\partial x_{\mp}}
$$

which may be easily verified for (7-8). Alternatively, we can parametrize the Fermi surface by the values of $p$ at the two intersections with the $x=$ constant line, $P_{ \pm}(x, t)$. Then the Euler equations take the form

$$
\partial_{t} P_{ \pm}=x-P_{ \pm} \partial_{x} P_{ \pm}
$$

In the remainder of this paper we will primarily discuss the original solutions (11) of [18].

\subsection{Collective Field Theory}

So far our discussion has been in the open string language of matrix quantum mechanics. From this point of view we have described some matrix configurations which are time dependent, but there has not been a notion of "space", and therefore no "cosmology" to discuss. The spatial coordinate $x$ is an emergent quantity which can be seen only after passing to the closed string description by introducing the density of eigenvalues, or the collective field

$$
\rho(x, t)=\sum_{i} \delta\left(x-x_{i}(t)\right)
$$

In the continuum limit it is convenient to trade the density $\rho(x, t)$ for a scalar field $\phi(x, t)$ through $\rho(x, t)=\partial_{x} \phi(x, t)$. The action of the field $\phi(x, t)$ is $[27,28]$

$$
S=\int d t d x\left[\frac{\left(\partial_{t} \phi\right)^{2}}{2 \partial_{x} \phi}-\frac{\pi^{2}}{6}\left(\partial_{x} \phi\right)^{3}+\left(\frac{1}{2} x^{2}-\mu\right) \partial_{x} \phi\right]+\Delta S,
$$

where $\Delta S$ is the singular term

$$
\Delta S=\frac{1}{2} \int d t d x \partial_{x} \phi\left[\partial_{x} \partial_{x^{\prime}} \log \left|x-x^{\prime}\right|\right]_{x=x^{\prime}},
$$

which is a part of the Jacobian of the change of variables (17) from eigenvalues $x_{i}(t)$ to the density $\rho(x, t)[27]$. The singular term $\Delta S$ contributes at higher order in the loop expansion parameter $g_{s}=\mu^{-1}$, but it will nevertheless play a central role in what follows.

Any distribution of eigenvalues corresponds to a definite state of the two dimensional collective field theory. However, for generic Fermi surfaces such a state cannot be described as a classical solution of the collective field theory because of the presence of folds or disconnected pieces $[29,30]$. In fact, it can represent states of the theory where quantum dispersions of fields are of the same order as their classical expectation values [31, 32]. However, for the time

dependent Fermi surfaces considered in this paper the profiles are quadratic - there are no 
folds - and such Fermi surfaces can indeed be represented as classical solutions of collective field theory.

The classical equation of motion following from the Lagrangean (18) is

$$
2 \partial_{t} \frac{\partial_{t} \phi}{\partial_{x} \phi}=\partial_{x}\left[\pi^{2}\left(\partial_{x} \phi\right)^{2}+\left(\frac{\partial_{t} \phi}{\partial_{x} \phi}\right)^{2}-\left(x^{2}-2 \mu\right)\right] .
$$

since we must ignore $\Delta S$ in the classical limit. The ansatz

$$
\partial_{x} \phi_{0}=\frac{1}{\pi} P_{0}(x, t), \quad \partial_{t} \phi_{0}=-\frac{1}{\pi} P_{0}(x, t) F(t),
$$

where

$$
P_{0}(x, t)=\sqrt{(x-\dot{F})^{2}-2 \mu},
$$

solves the equation of motion (20) for all functions $F(t)$. Imposing the consistency conditions $\partial_{t} \partial_{x} \phi_{0}=\partial_{x} \partial_{t} \phi_{0}$ on (21) we find

$$
\frac{d^{2} F(t)}{d t^{2}}=F(t)
$$

and so

$$
F(t)=\lambda_{-} e^{-t}-\lambda_{+} e^{t} .
$$

An alternative procedure that yields the solution (21) with (22) and (24) is to verify that the profiles of the Fermi surface

$$
P_{ \pm}(x, t)= \pm P_{0}(x, t)+F(t),
$$

satisfy the Euler equations (16).

The two parameter family of solutions to (23) given in (24) is identical to the matrix model solution (11) that was generated from the static solution $F=0$ by the action of $w_{01}$ and $w_{10}$. The explicit solutions for other $w_{0 s}, w_{r 0}$ solutions can be also obtained in principle, though they involve solutions of higher order algebraic equations.

The form of (22) restricts the $x<0$ branch of interest to

$$
x<-\sqrt{2 \mu}+\dot{F} .
$$

The solution generated by $w_{01}$ has $F(t)=\lambda_{-} e^{-t}$ so, as $t=-\infty$, the Fermi surface is pushed to the region of large negative $x$. In perturbation theory, the collective field therefore does not support excitations for any finite $x$ - there is no universe at $t=-\infty$. At finite times there is some allowed region of $x$ which is growing at $t$ increases so that, as $t \rightarrow \infty$, the Fermi surface becomes the static solution (2), which is the usual universe of the two dimensional string around the ground state. The entire solution thus represents the creation of a universe. In a similar way, solutions generated by $w_{10}$ represent the destruction of a universe. We will henceforth concentrate on universe destruction. 
It will be important for our considerations to introduce a lower bound $x_{\min }=-\Lambda$ in $(26)$ which restricts the universe to the finite volume

$$
-\Lambda<x<-\sqrt{2 \mu}+\dot{F}
$$

The origin of the infrared regulator is the potential $V(x)$ before performing the double scaling

limit. This leads to $\Lambda \sim \sqrt{\beta N} \sim 1 / \sqrt{\mu_{0}} \rightarrow \infty$. We should therefore think of the volume of the universe as a quantity of order $\sqrt{\beta N}$ in $x$ space.

The solutions of collective field theory which correspond to the more general solutions generated by $w_{0 s}$ or $w_{r 0}$ are more complicated to obtain, since these correspond to nonlinear transformations of the collective field. Nevertheless, as mentioned above, they can be obtained by solving higher order equations in terms of phase space variables and translating these solutions in the collective field language.

\subsection{Backgrounds in Matrix model}

What is the meaning of these cosmologies in the original holographic theory, i.e. matrix quantum mechanics? This is an interesting question because in this case the holographic theory has no "space" at all and these cosmologies are encoded as specific time dependent configurations of the matrix $M(t)$. The Hamiltonian for matrix quantum mechanics reads

$$
H_{M}=\operatorname{Tr}\left[\Pi_{M}^{2}-M^{2}\right]
$$

and the $W_{\infty}$ charges are given by

$$
w_{r s}=\frac{1}{2} e^{(r-s) t} \operatorname{Tr}\left[\left(M-\Pi_{M}\right)^{r}\left(M+\Pi_{M}\right)^{s}\right] .
$$

If $|\mu\rangle$ denotes the ground state of this system, a cosmological background generated by $w_{s 0}$ is denoted by

$$
|\lambda\rangle=\exp \left[i \lambda w_{s 0}\right]|\mu\rangle
$$

The expectation values of a typical invariant quantity may then be formally expressed as

$$
\langle\lambda|\mathcal{O}| \lambda\rangle=\left\langle\mu\left|\mathcal{O}^{\prime}\right| \mu\right\rangle
$$

where

$$
\mathcal{O}^{\prime}=e^{-i \lambda w_{s 0}} \mathcal{O} e^{i \lambda w_{s 0}}
$$

This computation is purely formal because the left hand side of eq. (31) is not meaningful because the state $|\lambda\rangle$ is not normalizable. States such as $|\lambda\rangle$ form a subset of the discrete states in the $c=1$ model [33]. These, it is believed, should not be really be regarded as states in the 
spectrum of the original model. Instead, they are interpreted as deformed backgrounds. The formal manipulation suggests that the appropriate deformed Hamiltonian is

$$
H^{\prime}=H_{M}+\lambda s e^{s t} \operatorname{Tr}\left(M-\Pi_{M}\right)^{s} .
$$

In other words, the holographic interpretation of the cosmological background is the modified matrix model whose Hamiltonian is $H^{\prime}$. This interpretation is similar to the proposed description of two dimensional black holes in terms a matrix model deformed by a $\operatorname{Tr} M^{-2}$ potential [34].

\subsection{Fluctuations}

The sole perturbative closed string excitation around an arbitrary classical background is given by the fluctuation of the collective field around the corresponding classical solution of collective field theory $\phi_{0}(x, t)$

$$
\phi(x, t)=\phi_{0}(x, t)+\frac{1}{\sqrt{\pi}} \eta(x, t) .
$$

In our applications we identify the field $\eta$ with the spacetime tachyon ${ }^{1}$. Inserting the expression (34) into the action (18) we find the quadratic action of the fluctuations

$$
S^{(2)}=\frac{1}{2} \int d x d t \frac{1}{\pi \partial_{x} \phi_{0}}\left[\left(\partial_{t} \eta\right)^{2}-\left(\left(\pi \partial_{x} \phi_{0}\right)^{2}-\left(\frac{\partial_{t} \phi_{0}}{\partial_{x} \phi_{0}}\right)^{2}\right)\left(\partial_{x} \eta\right)^{2}-2 \frac{\partial_{t} \phi_{0}}{\partial_{x} \phi_{0}} \partial_{t} \eta \partial_{x} \eta\right] .
$$

This form of the quadratic action is valid for fluctuations around any classical solution $\phi_{0}(x, t)$. It is useful to interpret the action in terms of a massless scalar field propagating in a spacetime with metric

$$
d s^{2}=-d t^{2}+\frac{\left(d x+\frac{\partial_{t} \phi_{0}}{\partial_{x} \phi_{0}} d t\right)^{2}}{\left(\pi \partial_{x} \phi_{0}\right)^{2}} .
$$

For the specific case of matrix cosmology (21) we have

$$
S^{(2)}=\frac{1}{2} \int d x d t \frac{1}{P_{0}(x, t)}\left[\left(\partial_{t} \eta\right)^{2}-\left(P_{0}(x, t)^{2}-F(t)^{2}\right)\left(\partial_{x} \eta\right)^{2}+2 F(t) \partial_{t} \eta \partial_{x} \eta\right]
$$

and the background metric becomes

$$
d s^{2}=-d t^{2}+\frac{(d x-F(t) d t)^{2}}{P_{0}(x, t)^{2}} .
$$

The coordinate $x$ takes values in the interval (26). At the end-points of this space the field $\eta(x, t)$ satisfies Dirichlet boundary conditions. This follows from the fact that the integral of the full collective field $\int d x \partial_{x} \phi$ is the total number of fermions and therefore fixed ${ }^{2}$.

\footnotetext{
${ }^{1}$ In general leg-pole factors must be taken into account. These result in a nonlocal redefinition of the field which will not be essential in our discussion.

${ }^{2}$ Strictly this argument implies only that $\eta\left(x_{\min }\right)-\eta(-\sqrt{2 \mu})=0$. That fermions are in fact prevented from leaking out in either end of the interval is clear prior to taking the double scaling limit, and this property is inherited by the scaled theory.
} 
At the classical level the action (37) is invariant under Weyl rescalings and so it determines the metric only up to an overall conformal factor. In (38) this factor was chosen so that spacetime is flat. This can be seen explicitly by transforming the spatial coordinate as

$$
x=-\sqrt{2 \mu} \cosh y+\dot{F}, \quad \tau=t,
$$

so that the metric becomes

$$
d s^{2}=-d t^{2}+d y^{2}
$$

and the boundary condition simply becomes a Dirichlet condition at $y=0$. In these coordiantes the problem thus returns to the static case.

In terms of the original coordinates $(x, t)$ we have a problem similar to the moving mirror problem $[1,35]$, with the mirror trajectory given by the upper limit of the coordinate range (26). In the moving mirror problem particle production can be entirely rephrased in terms of the anomalous transformation of the energy momentum tensor under the conformal transformation which makes the mirror stationary. The vacuum in static coordinates then appears as a collection of particles in the frame where the mirror moves. Particle production in matrix cosmology works similarly except that, as we shall see in section 4.2 , the vacuum is prescribed differently.

In usual quantum field theory there is, in the absence of special symmetries, no sense in which one vacuum is preferred over the other - the appropriate choice is determined by the nature of observers. This vacuum ambiguity enters concrete computations through the normal ordering prescription which is implemented at "equal time", a notion that depends on the observer or, more precisely, on the coordinate system. String theory is different because ultraviolet divergences are absent and therefore physical observables, such as the one loop free energy, are finite. The way this comes about in the collective field theory description is that the singular term (19) acts as a counterterm that cancels all divergences. The form of this singular term singles out a specific coordinate system, by regulating physical quantities at equal matrix time. This circumstance forces us to use the same time $t=\tau$ in the original $(x, t)$ coordinates and the static coordinates $(y, \tau)$.

\section{Particle Production in Matrix Cosmology}

In this section we discuss the causal structure of the metric seen by the fluctuations and define notions of in and out modes accordingly. We derive the nontrivial Bogolubov transform relating the in and out modes, and we determine the corresponding spectrum of particles. We consider for definiteness the destruction of a universe, i.e. a draining Fermi sea $\left(F=-\lambda e^{t}\right)$. 


\subsection{Causal Structure}

A general problem in the study of time-dependent backgrounds is the definition of proper physical observables. For example, it is often difficult to define an S-matrix, because there are no suitable in and out regions. One might expect that matrix cosmology would suffer from this problem, since the entire spacetime disappears at late times. However, as we discuss now, this is fortunately not the case.
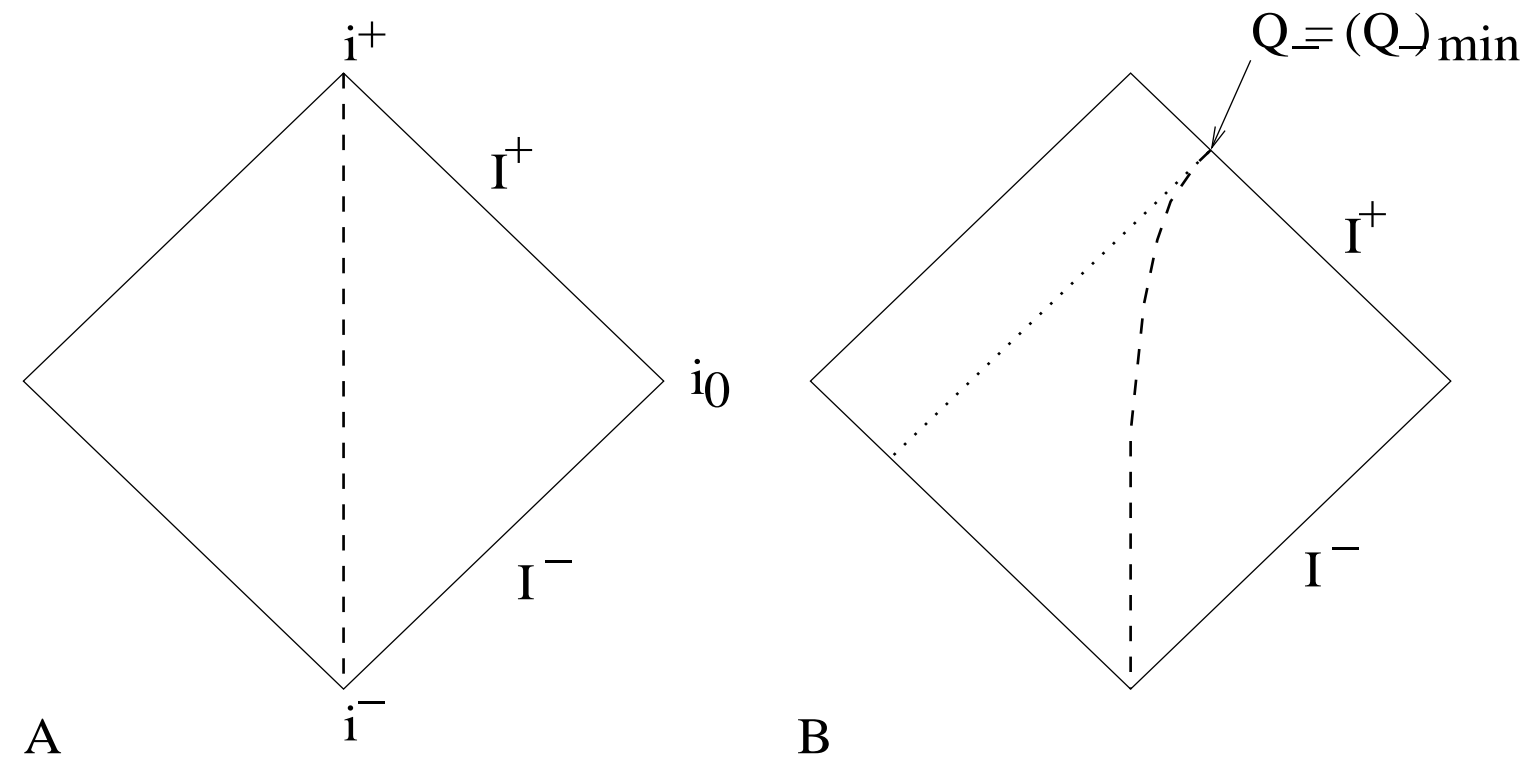

Figure 1: Causal structures in $(\mathrm{A})$ static $(y)$ and $(\mathrm{B})$ cosmological $(Q)$ coordinates. The dashed lines refer to the boundary of spacetime (the endpoint of the eigenvalue distribution) in the two different coordinates.

The most natural coordinate for spacetime processes is not the matrix model coordinate $x$, but rather the exponentiated coordinate $Q$ defined through ${ }^{3}$

$$
x=-\sqrt{2 \mu} \cosh Q
$$

We will think of $Q$ as the physical spacetime coordinate and refer to it as the cosmological coordinate. In our applications we will in addition find it essential to employ the static coordinate $y$ which transforms the matrix cosmology to Minkowski space (40) with a Dirichlet condition at $y=0$. According to (39) we have the relations

$$
\cosh y=\cosh Q+\frac{\dot{F}}{\sqrt{2 \mu}}=\cosh Q-\frac{\lambda}{\sqrt{2 \mu}} e^{t}
$$

\footnotetext{
${ }^{3}$ In the literature one often encounters the coordinate $q$ defined through $x=-e^{-q}$ (see $e . g$. [39]). Our $Q \simeq q$ at asymptotic distances but $Q$ has the advantage that the turning point of the Fermi sea is at $Q=0$.
} 
The time coordinate $t$ is the same for all these coordinate systems.

In the static coordinates $(y)$ a natural in region $\mathcal{I}^{-}$is defined by taking the light-cone coordinate $y_{-}=y-t \rightarrow \infty$. Similarly, a natural out region $\mathcal{I}^{+}$is defined by taking the lightcone coordinate $y_{+}=y+t \rightarrow \infty$. It is important to distinguish these regions from the future and past time-like infinities, $\imath^{ \pm}$, which are reached by taking $t \rightarrow \pm \infty$ at fixed $y$, and also from the space-like infinity, $\imath^{0}$, which is reached by taking $y \rightarrow+\infty$ at fixed time $t$. These are all indicated in figure $1(\mathrm{~A})$.

This discussion of asymptotic regions in the flat coordinates translates nicely into the cosmological coordinates $(Q)$. Indeed, define light-cone coordinates $Q_{ \pm}=Q \pm t$ and use (42) to obtain

$$
\begin{aligned}
Q_{ \pm} & =Q \pm t \\
& = \pm t+\cosh ^{-1}\left\{\cosh y+\frac{\lambda}{\sqrt{2 \mu}} e^{t}\right\} \\
& = \pm \frac{y_{+}-y_{-}}{2}+\cosh ^{-1}\left\{\cosh \left(\frac{y_{+}+y_{-}}{2}\right)+\frac{\lambda}{\sqrt{2 \mu}} e^{\frac{y_{+}-y_{-}}{2}}\right\}
\end{aligned}
$$

The in region $\mathcal{I}^{-}$is defined by taking $y_{-} \rightarrow \infty$ with $y_{+}$fixed so (43) gives

$$
Q_{ \pm}=y_{ \pm}+O\left(e^{-y_{-}}\right) \quad \text { on } \mathcal{I}^{-}
$$

The in region $\mathcal{I}^{-}$is thus defined in physical coordinates by taking $Q_{-} \rightarrow \infty$ with $Q_{+}$fixed. Of course this is rather obvious, since it is clear from (42) that the static coordinates $(y)$ agree with the cosmological coordinates $(Q)$ at early times. The point of making the notion of "early times" precise is to avoid confusion about the concept "late times" in the following.

The out region $\mathcal{I}^{+}$is defined by taking $y_{+} \rightarrow \infty$ with $y_{-}$fixed; and so (43) gives

$$
Q_{ \pm}=y_{ \pm}+\log \left(1+\tilde{\lambda} e^{-y_{-}}\right)+O\left(e^{-y_{+}}\right) \quad \text { on } \mathcal{I}^{+}
$$

where $\tilde{\lambda}=\sqrt{\frac{2}{\mu}} \lambda$. Thus $Q_{+} \rightarrow \infty$ with $Q_{-}$fixed will take one to the out region $\mathcal{I}^{+}$. The relation (45) is easily inverted on $\mathcal{I}^{+}$to find

$$
y_{ \pm}=Q_{ \pm}+\log \left(1-\tilde{\lambda} e^{-Q_{-}}\right)+O\left(e^{-Q_{+}}\right)
$$

An important feature of this expression is the branch cut at $Q_{-}=\log \tilde{\lambda}$. Thus we are reaching the out region by taking $Q_{+} \rightarrow \infty$ with $Q_{-}$fixed at some value larger than the minimal value $\left(Q_{-}\right)_{\min }=\log \tilde{\lambda}$. The significance of this limiting value is that the boundary of the draining Fermi sea asymptotically approaches the light-like trajectory parametrized by $Q_{-}=\left(Q_{-}\right)_{\min }$. This is indicated on Figure 1(B).

In summary, the disappearance of spacetime by definition prevents the presence of a future timelike infinite $\imath^{+}$. However, there is still a well-defined out region $\mathcal{I}^{+}$, defined by taking 
$Q_{+} \rightarrow \infty$ with $Q_{-}$fixed at some value larger than the asymptotic trajectory of the tachyon wall $\left(Q_{-}\right)_{\min }=\log \tilde{\lambda}$. This means it makes sense to discuss observables in the form of S-matrix elements.

\subsection{The in and out Modes}

The equation of motion for the fluctuating collective field $\eta$ can be solved exactly. This is most easily done in the static coordinates $(y)$ where the equation of motion is satisfied by simple plane waves. After taking the Dirichlet boundary condition at $y=0$ into account, an obvious basis of solutions is given by

$$
u_{\omega}^{\mathrm{in}}(y, t)=\frac{1}{\sqrt{\pi \omega}} e^{-i \omega t} \sin \omega y \quad(\omega>0)
$$

and their complex conjugates. The normalization of (47) has been chosen such that these modes form an orthonormal basis with respect to the Klein-Gordon norm

$$
\left(u_{\omega^{\prime}}, u_{\omega}\right)=i \int_{\Sigma} d \Sigma^{\mu}\left(u_{\omega} \partial_{\mu} u_{\omega^{\prime}}^{*}-u_{\omega^{\prime}}^{*} \partial_{\mu} u_{\omega}\right)=\delta\left(\omega-\omega^{\prime}\right)
$$

on any Cauchy surface $\Sigma$. We can find the exact modes in the physical coordinates $(Q)$ by solving (42) for $y$ in terms of $Q$ and $t$, and substituting the result into (47). The modes obtained in this way will in general have a complicated dependence on the time $t$, but this dependence become quite simple in the in and out regions.

In the in region $\left(\mathcal{I}^{-}\right)$the static coordinates $(y)$ coincide with the cosmological coordinates $(Q)$ and so we can write $(47)$ as

$$
u_{\omega}^{\text {in }}(Q, t)=\frac{1}{\sqrt{\pi \omega}} e^{-i \omega t} \sin \omega Q=\frac{i}{\sqrt{4 \pi \omega}} e^{i \omega Q_{+}} \quad \text { on } \mathcal{I}^{-}
$$

In the second equality we omitted the term depending on $Q^{-}$because, on $\mathcal{I}^{-}$, this term does not contribute to the probability current. Equivalently, the inner product (48) reduces on $\mathcal{I}^{-}$ to

$$
\left(u_{\omega^{\prime}}, u_{\omega}\right)=i \int d Q_{+}\left(u_{\omega} \partial_{Q_{+}} u_{\omega^{\prime}}^{*}-u_{\omega^{\prime}}^{*} \partial_{Q_{+}} u_{\omega}\right) \quad \text { on } \mathcal{I}^{-}
$$

According to (49) the modes (47) reduce to the standard positive frequency plane waves in the in region. It is for this reason that we have specified them from the outset by the superscript "in".

In the out region $\left(\mathcal{I}^{+}\right)$the static coordinate $(y)$ is related to the cosmological coordinate $(Q)$ through (46) and so the modes (47) take the form

$$
u_{\omega}^{\text {in }}(Q, t)=-\frac{i}{\sqrt{4 \pi \omega}} e^{i \omega Q_{-}}\left(1-\tilde{\lambda} e^{-Q_{-}}\right)^{i \omega} \quad \text { on } \mathcal{I}_{+}
$$


We have omitted the term depending on $Q_{+}$because this term does not contribute to the current in the out region ${ }^{4}$. The canonical modes (49) in the in region thus evolve to the more complicated modes (51) in the out region. The change from dependence on $Q_{+}$to dependence on $Q_{-}$is due to the reflecting boundary conditions on the field which turn left-movers into right movers.

Since the in modes are rather complicated in the out region it is natural to introduce a different basis which is simple there. The obvious choice is to consider a set of modes parametrized by $\omega>0$ and which, in the out region, reduce to the canonical form

$$
u_{\omega}^{\text {out }}(Q, t)=-\frac{i}{\sqrt{4 \pi \omega}} e^{i \omega Q_{-}} \quad \text { on } \mathcal{I}_{+}
$$

It is obvious that modes in fact exist that satisfy the equations of motion everywhere and reduce to this expression in the out region: the in-modes and their complex conjugates all satisfy the equations of motion and, in the out region, they take the form given in (51) which, when the complex conjugates are included, span all functions of $Q_{-}$. The nontrivial content of selecting the modes (54) is the implied notion of positive frequency $\omega>0$ which, after quantization, amounts to the introduction of a particle concept. Since spacetime is rapidly evolving at late times it is not obvious a priori that any such notion should even exist in the out region. However, in the present context, the underlying matrix model singles out a preferred time coordinate $t$. The proposed out modes (54) are uniquely determined by their canonical dependence on this time coordinate.

\subsection{The Bogolubov Transformation}

The in modes depend on both the out modes and their complex conjugates, as encoded in the Bogolubov transform,

$$
u_{\omega}^{\text {in }}\left(Q_{-}\right)=\int_{0}^{\infty} d \bar{\omega}\left[\alpha(\bar{\omega}, \omega) u_{\bar{\omega}}^{\text {out }}\left(Q_{-}\right)+\beta(\bar{\omega}, \omega)\left(u_{\bar{\omega}}^{\text {out }}\left(Q_{-}\right)\right)^{*}\right] .
$$

We want to compute the Bogolubov coefficients $\alpha(\bar{\omega}, \omega)$ and $\beta(\bar{\omega}, \omega)$. To do so we introduce the Fourier transform $F$ of the in mode through,

$$
u_{\omega}^{\text {in }}\left(Q_{-}\right)=\int_{-\infty}^{\infty} d \omega^{\prime} e^{i \omega^{\prime} Q_{-}} F\left(\omega, \omega^{\prime}\right)
$$

\footnotetext{
${ }^{4}$ This is clearest in the $y$-coordinates where the inner product on $\mathcal{I}^{+}$is written as in (50) with $d Q_{+} \rightarrow d y_{-}$ and $\partial_{Q_{+}} \rightarrow \partial_{y_{-}}$. If we wish to write the inner product on $\mathcal{I}^{+}$as an integral over $d Q_{-}$we must use the more complicated tangent derivative

where

$$
\left.\frac{\partial}{\partial Q_{-}}\right|_{y_{-}}=\partial_{Q_{-}}+\left.\frac{\partial Q_{+}}{\partial Q_{-}}\right|_{y_{+}} \partial_{Q_{+}}
$$

$$
\left.\frac{\partial Q_{+}}{\partial Q_{-}}\right|_{y_{+}}=\frac{\tilde{\lambda} e^{-Q_{-}}}{1-\tilde{\lambda} e^{Q_{-}}} .
$$


We must be a little careful when inverting this expansion, because $Q_{-}$has a semi-infinite range bounded below by the minimum value $\left(Q_{-}\right)_{\min }=\ln \tilde{\lambda}$. Accordingly we compute,

$$
\begin{aligned}
\int_{\ln \tilde{\lambda}}^{\infty} \frac{d Q_{-}}{2 \pi} e^{-i \bar{\omega} Q_{-}} u_{\omega}^{\mathrm{in}}\left(Q_{-}\right) & =\int_{\ln \tilde{\lambda}}^{\infty} \frac{d Q_{-}}{2 \pi} \int_{-\infty}^{\infty} d \omega^{\prime} e^{i\left(\omega^{\prime}-\bar{\omega}\right) Q_{-}} F\left(\omega, \omega^{\prime}\right) \\
& =i \int_{-\infty}^{\infty} \frac{d \omega^{\prime}}{2 \pi} \frac{F\left(\omega, \omega^{\prime}\right)}{\omega^{\prime}-\bar{\omega}} e^{i\left(\omega^{\prime}-\bar{\omega}\right) \ln \tilde{\lambda}} \\
& =F(\omega, \bar{\omega})
\end{aligned}
$$

In the first step we assumed $\operatorname{Im}(\bar{\omega})<0$, which is implied already on the left hand side to ensure convergence. In the next step we closed the $\omega^{\prime}$-contour in the lower part of the complex plane. Using (51) for the in-modes we find

$$
\begin{aligned}
F(\omega, \bar{\omega}) & =\int_{\ln \tilde{\lambda}}^{\infty} \frac{d Q_{-}}{2 \pi} e^{-i \bar{\omega} Q_{-}} u_{\omega}^{\mathrm{in}}\left(Q_{-}\right) \\
& =-\frac{i}{\sqrt{4 \pi \omega}} \int_{\ln \tilde{\lambda}}^{\infty} \frac{d Q_{-}}{2 \pi} e^{i(\omega-\bar{\omega}) Q_{-}}\left(1-\tilde{\lambda} e^{-Q_{-}}\right)^{i \omega} \\
& =-\frac{i}{\sqrt{4 \pi \omega}} \frac{\tilde{\lambda}^{i(\omega-\bar{\omega})}}{2 \pi} \int_{0}^{1} d z z^{-i(\omega-\bar{\omega})-1}(1-z)^{i \omega} \\
& =-\frac{i}{\sqrt{4 \pi \omega}} \frac{\tilde{\lambda}^{i(\omega-\bar{\omega})}}{2 \pi} B(i(\bar{\omega}-\omega), 1+i \omega) .
\end{aligned}
$$

We changed the integration variable to $z=\tilde{\lambda} e^{-Q_{-}}$. Comparing the definition of the Bogolubov transform (55) with the Fourier transform (56), and recalling the definition of out modes (54), we now find the Bogolubov coeffcients,

$$
\begin{aligned}
& \alpha(\bar{\omega}, \omega)=\frac{1}{2 \pi} \sqrt{\frac{\bar{\omega}}{\omega}} \tilde{\lambda}^{i(\omega-\bar{\omega})} B(i(\bar{\omega}-\omega), 1+i \omega), \\
& \beta(\bar{\omega}, \omega)=\frac{1}{2 \pi} \sqrt{\frac{\bar{\omega}}{\omega}} \tilde{\lambda}^{i(\omega+\bar{\omega})} B(-i(\bar{\omega}+\omega), 1+i \omega) .
\end{aligned}
$$

The non-vanishing of the $\beta(\bar{\omega}, \omega)$ is interpreted as particle production in the matrix cosmology. The creation and annihilation operators in the in and out vacua are defined by the expansions,

$$
\begin{aligned}
\eta(Q, t) & =\int_{0}^{\infty} d \omega\left[a_{\omega}^{\text {in }} u_{\omega}^{\text {in }}(Q, t)+a_{\omega}^{\text {in† }} u_{\omega}^{\text {in* }}(Q, t)\right] \\
& =\int_{0}^{\infty} d \omega\left[a_{\omega}^{\text {out }} u_{\omega}^{\text {out }}(Q, t)+a_{\omega}^{\text {out }} u_{\omega}^{\text {out } *}(Q, t)\right]
\end{aligned}
$$

and so, comparing with (55), one finds,

$$
a_{w}^{\text {out }}=\int_{0}^{\infty} d \bar{w}\left[\alpha(\omega, \bar{w}) a_{\bar{w}}^{i n}+\beta^{*}(\omega, \bar{w}) a_{\bar{w}}^{i n \dagger}\right] .
$$


The quantity that we want to compute is the expectation value of the out number operator in the in vacuum,

$$
\begin{aligned}
N(w) & =\left\langle a_{\omega}^{\text {out }} a_{\omega}^{\text {out }}\right\rangle_{\text {in }} \\
& =\int_{0}^{\infty} d \bar{w}|\beta(w, \bar{w})|^{2} \\
& =\int_{0}^{\infty} \frac{d \bar{w}}{4 \pi(\omega+\bar{w})} \frac{\sinh \pi \omega}{\sinh \pi(\omega+\bar{w}) \sinh \pi \bar{w}} .
\end{aligned}
$$

This is our final result for the particle spectrum on $\mathcal{I}^{+}$. The integral is convergent at high energies, as it should be. There is a logarithmic divergence for small $\bar{\omega}$ which is interpreted as a linear divergence in $Q_{-}$, i.e. it is the energy density which is finite.

\section{The Energy Momentum Tensor}

In this section we analyze the energy-momentum tensor of the fluctuations in the cosmological background. We begin by reviewing the static case and then turn to the time-dependent setting.

\subsection{The Static Case}

We begin the discussion by considering the static case $F=0$. The Hamiltonian for the fluctuations then simplifies to

$$
H=\int d x P_{0}\left[\frac{1}{2} \Pi_{\eta}^{2}+\frac{1}{2}\left(\partial_{x} \eta\right)^{2}\right]+\Delta H
$$

where

$$
\Delta H=\int d x P_{0}\left[\frac{1}{2 \pi} \partial_{x} \partial_{x^{\prime}} \log \left|x-x^{\prime}\right|\right]_{x=x^{\prime}},
$$

is the Hamiltonian form of (19). This last term is actually independent of the fluctuating field $\eta$, but it is included because it contributes at the same order in the loop expansion parameter $g_{s}=\mu^{-1}$ as the quadratic fluctuations. The measure $d x P_{0}$ that appear in both (64) and (65) translates in the underlying matrix model to $\operatorname{de} \rho(e)$, which is the obvious continuum version of the sum over eigenvalues. The expressions in the square brackets then correspond to the quantum fluctuations of the eigenvalues.

For explicit computations it is useful to employ the static coordinates $y$ where the metric for the fluctuation of the collective field is the simple Minkowskian (40), and the Dirichlet condition is imposed at $y=0$. In this coordinate system the Green's function takes the simple form

$$
D(\bar{t}, \bar{y} ; t, y)=-\frac{1}{4 \pi} \log \left(\frac{\Delta t^{2}-\Delta y^{2}}{\Delta t^{2}-(y+\bar{y})^{2}}\right),
$$

where $\Delta t=\bar{t}-t$ and $\Delta y=\bar{y}-y$. The denominator arises from the image charge that enforces the Dirichlet condition. The Green's function in other coordinate systems can be found by simply substituting expressions for $\tau$ and $y$ as functions of those other coordinates. 
The Green's function diverges at coincident points so, to do the calculations, we must regulate the theory. We interpret two point functions by using a cutoff in $x$ coordinates according to the prescription

$$
\left\langle\left(\partial_{x} \eta\right)^{2}\right\rangle=\lim _{\bar{x} \rightarrow x} \partial_{\bar{x}} \partial_{x} D(\bar{t}, \bar{y} ; t, y)
$$

where the point-splitting $\bar{x} \rightarrow x+\frac{\epsilon}{2} ; \quad x \rightarrow x-\frac{\epsilon}{2}$ is implied. The $t$ and $y$ (and their barred analogues) are functions of these slightly shifted $x$ (and $\bar{x}$ ). Evaluating the remaining expression we find

$$
\begin{aligned}
\left\langle\left(\partial_{x} \eta\right)^{2}\right\rangle & =-\frac{1}{2 \pi} \lim _{\bar{x} \rightarrow x}\left\{\frac{\bar{y}^{\prime} y^{\prime}}{(\bar{y}-y)^{2}}+\frac{\bar{y}^{\prime} y^{\prime}}{(\bar{y}+y)^{2}}\right\}, \\
& =-\frac{1}{2 \pi \epsilon^{2}}-\frac{1}{8 \pi}\left(\frac{y^{\prime}}{y}\right)^{2}-\frac{1}{12 \pi}\{y, x\}+\mathcal{O}\left(\epsilon^{2}\right),
\end{aligned}
$$

where the limit $\epsilon \rightarrow 0$ is implied and we introduced the Schwarzian derivative

$$
\{y, x\}=\frac{y^{\prime \prime \prime}}{y^{\prime}}-\frac{3}{2}\left(\frac{y^{\prime \prime}}{y^{\prime}}\right)^{2}
$$

as well as the notations $y^{\prime}=\partial_{x} y=-1 / P_{0}$ etc. (and similarly for $\bar{y}$ and $\bar{x}$ ).

The canonical momentum is given by the Hamiltonian equation of motion as

$$
\Pi_{\eta}=\frac{1}{P_{0}} \partial_{t} \eta
$$

Using this expression, it is straightforward to compute the two point correlator of the momenta using the Green's function (66) and the point-splitting procedure already used in (67). The result is

$$
\left\langle\Pi_{\eta}^{2}\right\rangle=-\frac{1}{2 \pi \epsilon^{2}}+\frac{1}{8 \pi}\left(\frac{y^{\prime}}{y}\right)^{2}-\frac{1}{12 \pi}\{y, x\}+\mathcal{O}\left(\epsilon^{2}\right) .
$$

This result agrees precisely with (68), except for the sign of the second term, which is the part that arises from the images. Indeed, the computations leading to the two results are almost identical because, after taking the $P_{0}$ appearing in the denominator of $(70)$ into account, temporal derivatives act on the short distance part of the Green's function in the same way as spatial derivatives. This agreement between the potential energy (68) and the kinetic energy (71) is referred to as the virial theorem. The origin of the virial theorem in the matrix theory is the simple oscillator form of the potential after double scaling.

Collecting the results we find that the divergent pieces cancel and the final result for the expectation value is

$$
H_{\mathrm{gs}}=-\int d x P_{0} \frac{1}{12 \pi}\{y, x\}
$$

of the Hamiltonian (64) in the ground state. The explicit form (39) (with $F=0$ ) of $y(x)$ gives

$$
\{y, x\}=\frac{1}{P_{0}^{2}}\left(\frac{1}{2}+\frac{3 \mu}{x^{2}-2 \mu}\right) \rightarrow \frac{1}{2 P_{0}^{2}},
$$


for large $x^{2}$. The extensive part of the energy is therefore

$$
H_{\mathrm{gs}}=-\frac{1}{24 \pi}\left|\ln \left(-x_{\mathrm{min}}\right)\right|=\frac{1}{48 \pi} \ln \mu_{0}
$$

since $\left|x_{\min }\right|=\Lambda \sim 1 / \sqrt{\mu_{0}}$. This result is interesting for several reasons:

1. The singular term from the Jacobian (65) cancels the regularized singularities from the two-point functions and so the final result is finite, without the need for futher renormalization of the collective field theory ${ }^{5}$. This explicit understanding of how the matrix theory induces the correct counterterms in collective effective field theory is the origin of a preferred vacuum in the theory.

2. The image in the Green's function (66) contributes to the finite part of the two-point functions (68) and (71), but these contributions cancel in the total energy. It is not surprising that boundary conditions are unimportant for the extensive part of the energy in the thermodynamic limit; but it is nice to see how it works explicitly.

3. The finite part agrees with the one-loop result found by solving the matrix model explicitly $[36]^{6}$. This gives great confidence that we have interpreted the theory correctly.

Let us also mention the further results:

1. In collective field theory, the ground state of the matrix model is the vacuum defined in terms of the mode expansion of the fluctuation field in terms of modes $e^{ \pm i \omega t} \sin (\omega y)$ since this is the vacuum which leads to the two point function used above.

2. The presence of a finite ground state energy in this theory is an important signature that we are dealing with a string theory. In fact, at finite temperature $T$ this term, when added to the standard thermodynamic contribution, leads to a T-dual answer symmetric under $\pi T \rightarrow \frac{1}{\pi T}$ characteristic of string theory [15].

3. We have performed the calculation using a point splitting regulator in $x$ space. If we use instead a regulator in $y$ space, the Schwarzian derivative term will come from $\Delta H$. The divergences cancel as before leading to the same finite answer.

\footnotetext{
${ }^{5}$ This cancellation has been known from early days of collective field theory [15, 28, 37]. However, generally the image term has been ignored in these computations and our explicit verification of the virial theorem in the presence of regularization is also new, to the best of our knowledge.

${ }^{6}$ The energy (74) agrees with eq. 3.30 of [38] after dividing the result given there by two, because we only compute the energy of the $x<0$ part of the Fermi sea.
} 


\subsection{The EM-tensor in Matrix Cosmology}

Let us now generalize these considerations to matrix cosmology. As we have emphasized, matrix cosmology is related to the static case by a simple coordinate transformation which, near the asymptotic null infinity $\mathcal{I}^{+}$, takes the form

$$
y_{-}=Q_{-}+\log \left[1-\tilde{\lambda} e^{-Q_{-}}\right]+\mathcal{O}\left(e^{-Q_{+}}\right)
$$

as $Q_{+} \rightarrow \infty$ with $Q_{-}$fixed at some $Q_{-}>\ln \tilde{\lambda}$. As in previous sections we use the notation $y_{ \pm}=y \pm t$ and $Q_{ \pm}=Q \pm t$ for the static and cosmological light-cone coordinates and we also introduced the abbreviation $\tilde{\lambda}=\lambda \sqrt{\frac{2}{\mu}}$ to parametrize the limiting value $\left(Q_{-}\right)_{\min }=\ln \tilde{\lambda}$.

The static coordinates $(y)$ play a several roles in the problem. First, the cosmological coordinates $(Q)$ reduce to the static coordinates at early times; so the static coordinates define the in vacuum. Second, the Green's function (66) in static coordinates transform simply into the cosmological coordinates, since the transformation (75) is conformal (it does not mix the "+ " and " - " light-cone coordinates). This will allow us to use conformal techniques to study the problem at asymptotic null infinity $\mathcal{I}^{+}$. Following the standard strategy, we begin the discussion by writing the results from the static case in a form that transforms naturally under the conformal group.

The extensive part of the energy was computed in the previous subsection with the result (74). The volume factor in the static frame $(y)$ is related to the infrared cutoff in the matrix model coordinates $(x)$ through $x \sim e^{y}$ so we can express this result concisely in terms of the energy density

$$
\epsilon=-\frac{1}{48 \pi}
$$

in the static frame. Note that this formula is exact at $\mathcal{I}^{+}$since the subleading terms in (73) do in fact vanish as $Q_{+} \rightarrow \infty$ with $Q_{-}$fixed. The classical expression for the pressure density is identical to that of the energy density so, repeating the arguments in the previous subsection, we find $p=\epsilon$. The complete energy-momentum tensor in the static frame thus takes the form

$$
\left\langle T_{y_{+}, y_{+}}\right\rangle=\left\langle T_{y_{-}, y_{-}}\right\rangle=-\frac{1}{48 \pi}
$$

in light-cone coordinates

Let us now transform to the cosmological coordinates $Q$ which contain the behavior at late times. Since (75) is a conformal transformation we can use the standard, anomalous, transformation rule

$$
T_{Q_{-}, Q_{-}}=\left(\frac{\partial y_{-}}{\partial Q_{-}}\right)^{2} T_{y_{-}, y_{-}}+\frac{1}{24 \pi}\left\{y_{-}, Q_{-}\right\}_{S},
$$

of the EM-tensor. The second term is present because the short distance singularity of the EMtensor, although still cancelled by the explicit counter-term (65) in the collective field theory, 
differs by finite amounts in the two coordinate systems. The expectation value of (78) relates the EM-tensor of the static and the cosmological coordinates. Evaluating the derivatives we find

$$
\begin{aligned}
\frac{\partial y_{-}}{\partial Q_{-}} & =\frac{1}{1-\tilde{\lambda} e^{-Q_{-}}} \\
\left\{y_{-}, Q_{-}\right\}_{S} & =\frac{\tilde{\lambda} e^{-Q_{-}}\left(1-\frac{1}{2} \tilde{\lambda} e^{-Q_{-}}\right)}{\left(1-\tilde{\lambda} e^{-Q_{-}}\right)^{2}}
\end{aligned}
$$

and then (78) becomes simply

$$
\left\langle T_{Q_{-}, Q_{-}}\right\rangle=-\frac{1}{48 \pi}
$$

This means physical observers will experience no outgoing flux of energy at $\mathcal{I}^{+}$. This result is a tremendous surprise.

In standard computations, such as those considering moving mirrors, the static EM-tensor $\left\langle T_{y_{-}, y_{-}}\right\rangle$would be taken to vanish, and so only the second term (the Schwarzian derivative) in (78) would contribute. The associated energy would be interpreted as the energy of the particles produced by the expansion. This is also the natural interpretation of the Schwarzian derivative here. However, in the present context the particle production is partially obscured by another effect which is operative as well: the (one-loop) energy density of the "in" vacuum is non-zero and finite in the co-moving frame $(y)$. The corresponding EM-tensor is blueshifted in the frame of asymptotic observers $(Q)$ on $\mathcal{I}^{+}$and this leads to an energy flux. This effect is the origin of the first term in (78). Our remarkable result is that these two effects cancel each other precisely such that the cosmological vacuum has precisely the same EM-tensor as the static vacuum. This is very different from the standard computations.

The EM-tensor (81) takes the same value along the entire $\mathcal{I}^{+}$as it does on $\mathcal{I}^{-}$. This suggests that, despite appearances, $\mathcal{I}^{+}$is a perfectly nice locus to define large classes of observables. One may consider the scattering of particles from $\mathcal{I}^{-}$to $\mathcal{I}^{+}$, and correlation functions on $\mathcal{I}^{+}$itself. The existence of such observables cannot be taken for granted in a time-dependent setting but, in matrix cosmology, it would seem that they both exist and are computable. It would clearly be interesting to make this more explicit.

Our result that the one-loop energy of the matrix cosmology agrees with that of the static ground state is all the more surprising because the classical energy in this specific background does receive the very specific time-dependent contribution

$$
E_{\mathrm{cl}}=E_{\mathrm{cl}}^{(0)}-\dot{F} \int_{-\Lambda}^{-\sqrt{2 \mu}} d x x \sqrt{x^{2}-2 \mu}
$$

where $E_{\mathrm{cl}}^{(0)}$ is the static ground state energy. This is consistent with (33). That the one-loop energy is the same as in the ground state of course means there is no energy flux. 
In summary, we have found a remarkable cancellation between two apparently different contributions to the EM-tensor. The consequence is that the outgoing EM-tensor $T_{--}$is identical in the in and out vacua. This is quite unusual and surprising, at least to us. It is natural to suspect that the cancellation ultimately stems from the integrable nature of the underlying matrix theory. To understand this better, it would be interesting to make the formal description of the matrix cosmology as a deformed matrix model more concrete. It would also be interesting to extend the computation of the EM-tensor to the broader class of cosmological solutions generated in section 2.2 from the $W_{\infty}$-symmetry of the model.

\section{Acknowledgements}

We thank A. Jevicki, J. Karczmarek, H. Liu, L. Susskind and T. Takayanagi for discussions. PM would also like to thank A. Dhar, D. Ghoshal, R. Gopakumar, D. Jatkar, G. Mandal, S. Minwalla, S. Mukhi, S. Naik, A. Sen and L. Sriramkumar for useful discussions. A preliminary version of this work was presented by P.M. in the National Workshop on String Theory, December 2003 held at the Indian Institute of Technology, Kanpur, India. SRD and PM both thank the MCTP for hospitality during phases of this work. This work of J.D. and F.L. was supported in part by the Department of Energy under Grant No. DE-FG02-95ER40899. The work of S.R.D. and P.M. was supported by National Science Foundation grant PHY-0244811 and the Department of Energy grant No. DE-FG01-00ER45832.

\section{References}

[1] N. D. Birrell and P. C. W. Davies, "Quantum Fields In Curved Space", Cambridge University Press (1982).

[2] S. W. Hawking, "Particle Creation By Black Holes", Commun. Math. Phys. 43 (1975) 199.

[3] A. Strominger and C. Vafa, "Microscopic Origin of the Bekenstein-Hawking Entropy", Phys. Lett. B 379, 99 (1996) [arXiv:hep-th/9601029].

[4] C. G. Callan and J. M. Maldacena, "D-brane Approach to Black Hole Quantum Mechanics", Nucl. Phys. B 472, 591 (1996) [arXiv:hep-th/9602043].

[5] G. T. Horowitz and A. Strominger, Phys. Rev. Lett. 77, 2368 (1996) [arXiv:hepth/9602051].

[6] S. R. Das and S. D. Mathur, "Comparing decay rates for black holes and D-branes", Nucl. Phys. B 478, 561 (1996) [arXiv:hep-th/9606185]. 
[7] J. M. Maldacena, "The large N limit of superconformal field theories and supergravity", Adv. Theor. Math. Phys. 2, 231 (1998) [Int. J. Theor. Phys. 38, 1113 (1999)] [arXiv:hepth/9711200].

[8] E. Witten, "Anti-de Sitter space and holography", Adv. Theor. Math. Phys. 2, 253 (1998) [arXiv:hep-th/9802150].

[9] S. S. Gubser, I. R. Klebanov and A. M. Polyakov, "Gauge theory correlators from noncritical string theory", Phys. Lett. B 428, 105 (1998) [arXiv:hep-th/9802109].

[10] E. Witten, "Anti-de Sitter space, thermal phase transition, and confinement in gauge theories", Adv. Theor. Math. Phys. 2, 505 (1998) [arXiv:hep-th/9803131].

[11] A. Dhar, G. Mandal and S. R. Wadia, "Absorption vs decay of black holes in string theory and T-symmetry", Phys. Lett. B 388, 51 (1996) [arXiv:hep-th/9605234].

[12] J. M. Maldacena and A. Strominger, "Black hole greybody factors and D-brane spectroscopy", Phys. Rev. D 55, 861 (1997) [arXiv:hep-th/9609026].

[13] J. McGreevy and H. Verlinde, "Strings from tachyons: The c $=1$ matrix reloaded", JHEP 0312, 054 (2003) [arXiv:hep-th/0304224].

[14] I. R. Klebanov, J. Maldacena and N. Seiberg, "D-brane decay in two-dimensional string theory", JHEP 0307, 045 (2003) [arXiv:hep-th/0305159].

[15] S. R. Das and A. Jevicki, "String Field Theory And Physical Interpretation Of D = 1 Strings", Mod. Phys. Lett. A 5, 1639 (1990).

[16] J. Polchinski, Nucl. Phys. B 346, 253 (1990).

[17] A. M. Sengupta and S. R. Wadia, Int. J. Mod. Phys. A 6, 1961 (1991).

[18] J. L. Karczmarek and A. Strominger, "Matrix cosmology", [arXiv:hep-th/0309138].

[19] D. Minic, J. Polchinski and Z. Yang, "Translation Invariant Backgrounds In (1+1)Dimensional String Theory", Nucl. Phys. B 369, 324 (1992).

[20] G. W. Moore and R. Plesser, "Classical scattering in (1+1)-dimensional string theory", Phys. Rev. D 46, 1730 (1992) [arXiv:hep-th/9203060].

[21] S. Y. Alexandrov, V. A. Kazakov and I. K. Kostov, "Time-dependent backgrounds of 2D string theory", Nucl. Phys. B 640, 119 (2002) [arXiv:hep-th/0205079]. 
[22] J. L. Karczmarek and A. Strominger, "Closed string tachyon condensation at $\mathrm{c}=1$ ", [arXiv:hep-th/0403169].

[23] T. Takayanagi and N. Toumbas, "A matrix model dual of type 0B string theory in two dimensions", JHEP 0307, 064 (2003) [arXiv:hep-th/0307083]; M. R. Douglas, I. R. Klebanov, D. Kutasov, J. Maldacena, E. Martinec and N. Seiberg, "A new hat for the c $=1$ matrix model", [arXiv:hep-th/0307195].

[24] J. Avan and A. Jevicki, "Classical integrability and higher symmetries of collective string field theory", Phys. Lett. B 266, 35 (1991); J. Avan and A. Jevicki, "Quantum integrability and exact eigenstates of the collective string field theory", Phys. Lett. B 272, 17 (1991)

[25] S. R. Das, A. Dhar, G. Mandal and S. R. Wadia, "Gauge theory formulation of the C = 1 matrix model: Symmetries and discrete states", Int. J. Mod. Phys. A 7, 5165 (1992) [arXiv:hep-th/9110021]; S. R. Das, A. Dhar, G. Mandal and S. R. Wadia, "Bosonization of nonrelativistic fermions and W infinity algebra", Mod. Phys. Lett. A 7, 71 (1992) [arXiv:hep-th/9111021].

[26] E. Witten, "Ground ring of two-dimensional string theory", Nucl. Phys. B 373, 187 (1992) [arXiv:hep-th/9108004].

[27] A. Jevicki and B. Sakita, "The Quantum Collective Field Method And Its Application To The Planar Limit", Nucl. Phys. B 165, 511 (1980).

[28] A. Jevicki, "Matrix Models and Field Theory", Brown Preprint BROWN-HET-771, October 1990.

[29] J. Polchinski, Nucl. Phys. B 362, 125 (1991).

[30] A. Dhar, G. Mandal and S. R. Wadia, Int. J. Mod. Phys. A 8, 3811 (1993) [arXiv:hepth/9212027].

[31] S. R. Das and S. D. Mathur, Phys. Lett. B 365, 79 (1996) [arXiv:hep-th/9507141].

[32] S. R. Das, "D branes in 2d string theory and classical limits", [arXiv:hep-th/0401067].

[33] D. J. Gross, I. R. Klebanov and M. J. Newman, "The Two Point Correlation Function Of The One-Dimensional Matrix Model", Nucl. Phys. B 350, 621 (1991) A. M. Polyakov, "Selftuning Fields And Resonant Correlations In 2-D Gravity", Mod. Phys. Lett. A 6, 635 (1991); J. Avan and A. Jevicki in [24]

[34] A. Jevicki and T. Yoneya, "A Deformed matrix model and the black hole background in two-dimensional string theory," Nucl. Phys. B 411, 64 (1994) [arXiv:hep-th/9305109]. 
[35] R. D. Carlitz and R. S. Willey, "Reflections On Moving Mirrors", Phys. Rev. D 36, 2327 (1987).

[36] D. J. Gross and N. Miljkovic, "A Nonperturbative Solution Of D = 1 String Theory", Phys. Lett. B 238, 217 (1990); E. Brezin, V. A. Kazakov and A. B. Zamolodchikov, "Scaling Violation In A Field Theory Of Closed Strings In One Physical Dimension", Nucl. Phys. B 338, 673 (1990); C. Crnkovic, P. Ginsparg and G. W. Moore, "The Ising Model, The Yang-Lee Edge Singularity, And 2-D Quantum Gravity", Phys. Lett. B 237, 196 (1990).

[37] I. Andric and V. Bardek, Phys. Rev. D 32, 1025 (1985).

[38] I. R. Klebanov, "String theory in two-dimensions", [arXiv:hep-th/9108019].

[39] J. Polchinski, "What is string theory?", [arXiv:hep-th/9411028]. 